\title{
Estudo dos Parâmetros do Processo de Soldagem a Ponto por Fricção de Chapa Fina da Liga de Alumínio AA2198-T8
}

\author{
Camila Caroline de Castro ${ }^{1}$, Athos Henrique Plaine ${ }^{1}$, Nelson Guedes de Alcântara ${ }^{1}$, Jorge Fernandez dos Santos ${ }^{2}$ \\ ${ }^{1}$ Universidade Federal de São Carlos - UFSCar, Departamento de Engenharia de Materiais - DEMa, São Carlos, SP, Brasil. \\ ${ }^{2}$ Helmholtz Zentrum Geesthacht - HZG, Geesthacht, Schleswig-Holstein, Alemanha.
}

Recebido: 31 Ago., 2018

Aceito: 30 Out. 2018

E-mail(s): camila.ccastro@outlook.com (CCC)
Resumo: O presente trabalho consiste na investigação dos parâmetros de soldagem a ponto por friç̧ão (FSpW) - velocidade de rotação (VR), tempo de soldagem (TS) e profundidade de penetração (PP) - de chapas de AA2198-T8 com 1,6 mm de espessura. O estudo foi realizado com base em ensaios mecânicos de cisalhamento das juntas sobrepostas soldadas nas condições indicadas pelo método Taguchi, visando à determinação da condição ótima de soldagem do ponto de vista de propriedades mecânicas e de processo. Os resultados obtidos indicaram que todas as condições de soldagem avaliadas excedem o limite de carga em cisalhamento mínimo requerido para aplicação aeronáutica. A análise também apontou que as variáveis VR e PP são responsáveis por quase $90 \%$ da variação da carga de cisalhamento das juntas, enquanto a contribuição do TS mostrou-se consideravelmente menos significativa. Por fim, a condição ótima de soldagem foi encontrada para os valores mais altos de VR e PP, considerando-se os intervalos dos parâmetros empregados no estudo.

Palavras-chave: Soldagem a ponto por fricção; FSpW; AA2198-T8; método Taguchi.

\section{Study of the Friction Spot Welding Parameters of AA2198-T8 Thin Sheets}

\begin{abstract}
This work features the investigation of the refill friction stir spot welding (RFSSW) parameters - rotational speed (RS), welding time (WT) and plunge depth (PD) - of AA2198-T8 1.6-mm thick sheets. The study was performed based on lap shear strength tests of overlapped joints welded under conditions indicated by the Taguchi method in order to determinate the optimized combination of parameters, considering the mechanical properties and process conditions. Results based on mechanical testing indicate that all the welding conditions evaluated in the current study exceed the minimum criterion for lap shear strength required for aeronautic applications. RS and PD have shown to be responsible altogether for almost $90 \%$ of the welds' lap shear strength variation, while TS presents considerable less significant contribution to the joints' resistance. The optimized welding condition was found for highest RS and PD levels and lowest TS.
\end{abstract}

Key-words: Refill friction stir spot welding; FSpW; AA2198-T8; Taguchi method.

\section{Introdução}

O processo de soldagem a ponto por fricção (do inglês Refill Friction Stir Spot Welding (RFSSW) ou Friction Spot Welding (FSpW)) é uma técnica de união similar ou dissimilar de materiais leves no estado sólido, tais como alumínio, magnésio titânio e termoplásticos [1], patenteada pelo Helmholtz-Zentrum Geesthacht (HZG, antigo GKSS) que vem sendo desenvolvida desde os anos 2000. Diferentemente de outros processos de soldagem a ponto no estado sólido, como a soldagem por fricção por ponto (Friction Stir Spot Welding, FSSW), as juntas realizadas através do FSpW não apresentam o furo residual característico dessas técnicas, graças ao desenvolvimento de um dispositivo composto por três peças independentes (pino, camisa e anel de fixação, apresentados esquematicamente na Figura 1) que garante o preenchimento do furo deixado pela penetração da ferramenta na chapa.

O processo de soldagem a ponto por fricção é dividido em quatro etapas, e a variante do processo com a penetração da camisa é ilustrada na Figura 2. Na primeira delas, a ferramenta se aproxima da superfície do material a ser soldado e a força de fixação é aplicada pelo anel, de modo a garantir a imobilização das chapas durante o processo. O próximo passo consiste na penetração das chapas pela camisa, que gera um deslocamento de material plasticizado para a cavidade criada através do deslocamento
Este e um artigo publicado em acesso aberto (Open Access) que permite uso, distribuição e reprodução em qualquer meio, sem restriç̄es desde que sem fins comerciais e que 0 trabalho original seja corretamente citado. 


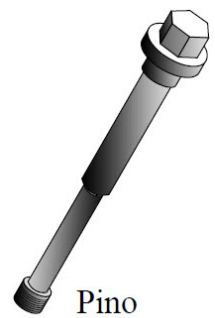

Pino

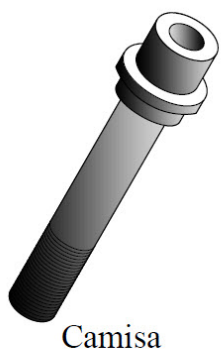

Camisa

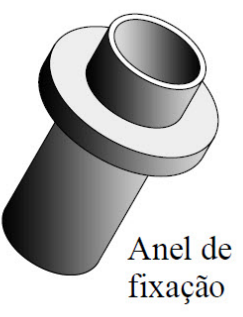

Montagem da ferramenta

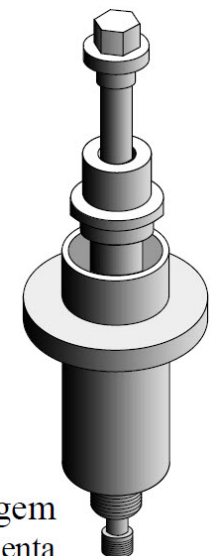

Figura 1. Ferramenta do processo de soldagem a ponto por friç̧ão. Adaptado de Lage [2].
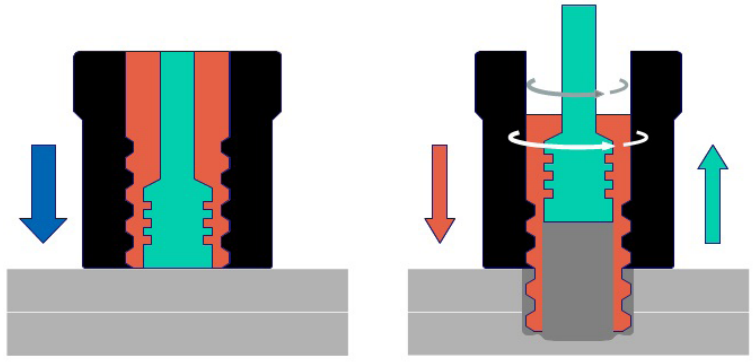

Anel de fixação

\section{(1)}
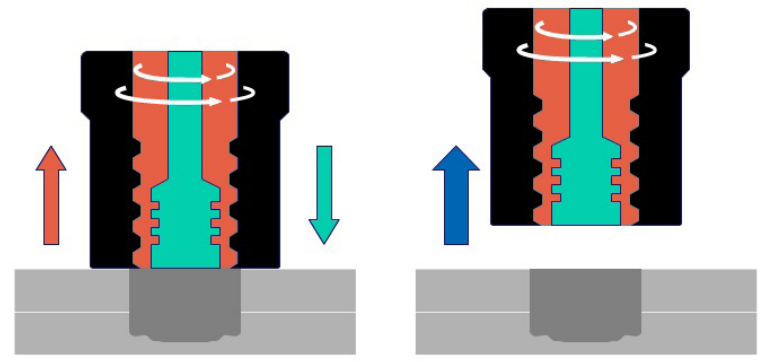

Figura 2. Soldagem a ponto por friç̧ão (FSpW): etapas do processo.

do pino na direção oposta à de penetração. Além do movimento no eixo axial, o pino e a camisa também são rotacionados a fim de aportar calor ao material através da friç̧ão, o que diminui a viscosidade e possibilita a mistura dos materiais da chapa superior e inferior, responsável pela união das partes. Durante a terceira etapa, as duas ferramentas rotativas são deslocadas nas direções opostas às da etapa de penetração, de modo que o pino empurre o material deslocado de volta ao interior da chapa e preencha o vazio deixado pela retração da camisa. Finalmente, no último estágio, a força aplicada pelo anel de fixação é liberada, e a ferramenta completa é removida da superfície da chapa, concluindo o processo de soldagem das duas chapas sobrepostas.

A liga AA2198-T8, classificada no sistema Al-Li, foi desenvolvida em 2002 pela Reynolds Metal Company e McCook Metals [3]. Visando à redução do peso de estruturas e menor consumo de combustíveis, ligas leves de alumínio têm sido amplamente utilizadas na indústria aeronáutica e automobilística uma vez que possuem resistência específica (i.e. relação entre resistência mecânica e densidade) adequada para a aplicação [3,4]. O desenvolvimento recente das ligas do sistema Al-Li viabilizaram a redução da densidade das ligas Al-Cu de em média 3\%, o que foi possível pela adição de $1 \%$ de lítio - metal com baixíssima densidade $\left(0,534 \mathrm{~g} / \mathrm{cm}^{3}\right)$ e altamente solúvel em Al. Além disso, as adições de Li modificam as propriedades mecânicas das ligas, como o módulo de Young (adições de $1 \%$ causam $6 \%$ ), dureza (formação de precipitados) e aumento da resistência à fadiga [3].

Atualmente, a maioria das uniões a ponto de componentes em estruturas aeronáuticas é realizada por fixação mecânica (como rebitamento, por exemplo) ou processos de soldagem convencionais que envolvem a fusão do material, tais como soldagem a ponto por resistência (RSW) e soldagem a ponto por laser (LSW) [5]. O RFSSW apresenta-se como uma excelente alternativa para a junção de chapas sobrepostas para aplicações na indústria aeronáutica, uma vez que não envolve a adição de materiais intrínseca às técnicas de união por fixação mecânica, 
resultando em estruturas mais leves [6]. Além disso, por ser uma técnica de união no estado sólido, defeitos associados a processos de soldagem convencionais por fusão são evitados, o que é especialmente interessante para a soldagem de ligas de alumínio de alta resistência - consideradas insoldáveis por estes métodos $[1,7]$.

O Brasil é líder mundial na produção de jatos comerciais para até 150 passageiros, além de figurar entre os maiores fabricantes globais de jatos executivos. Dados disponibilizados pela Embraer (sumarizados na Figura 3) mostram que na última década a companhia produziu quase 2200 unidades de aviões comerciais e executivos, além de aeronaves complexas para aplicação no setor de defesa \& segurança. Além dos números operacionais, a Figura 4 mostra a tendência expressiva de crescimento da receita líquida da empresa nos últimos quatro anos, cujo faturamento na última década ultrapassou R\$ 143 bilhões, dos quais R\$ 18,7 referem-se ao ano de 2017 [8].

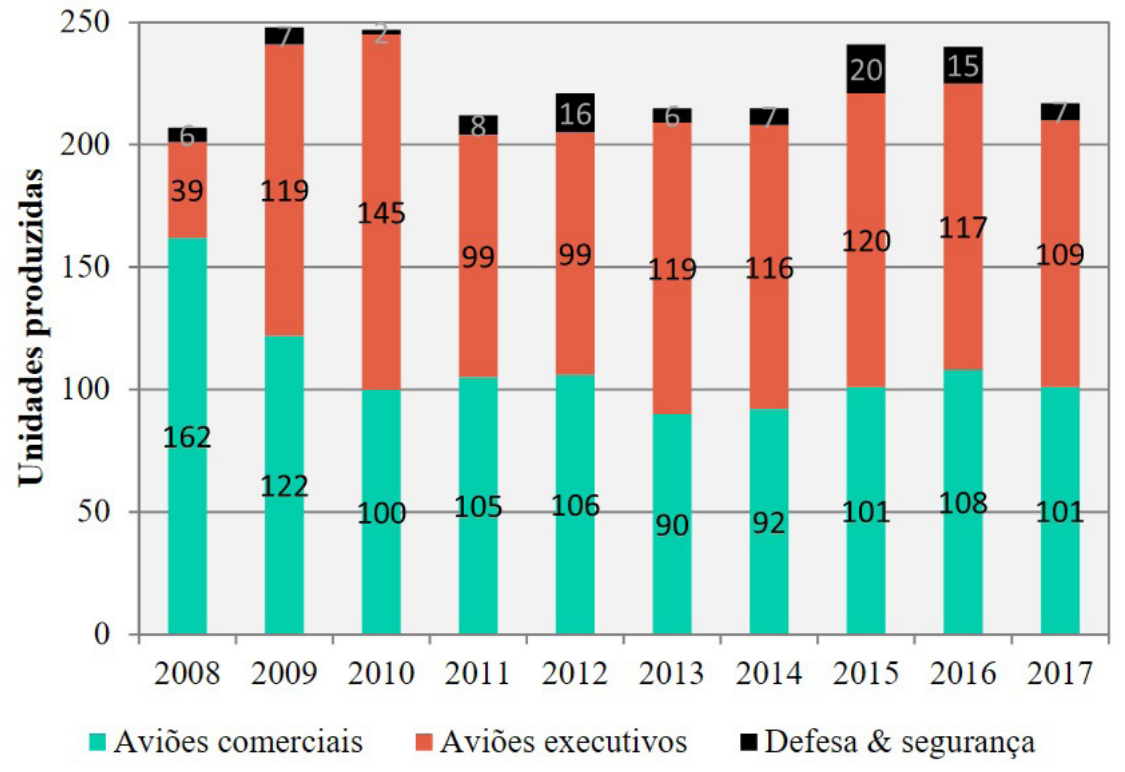

Figura 3. Operação da Embraer: número de aeronaves produzidas ao longo da última década. Adaptado de: Relatórios Anuais de 2008-2017 [8].

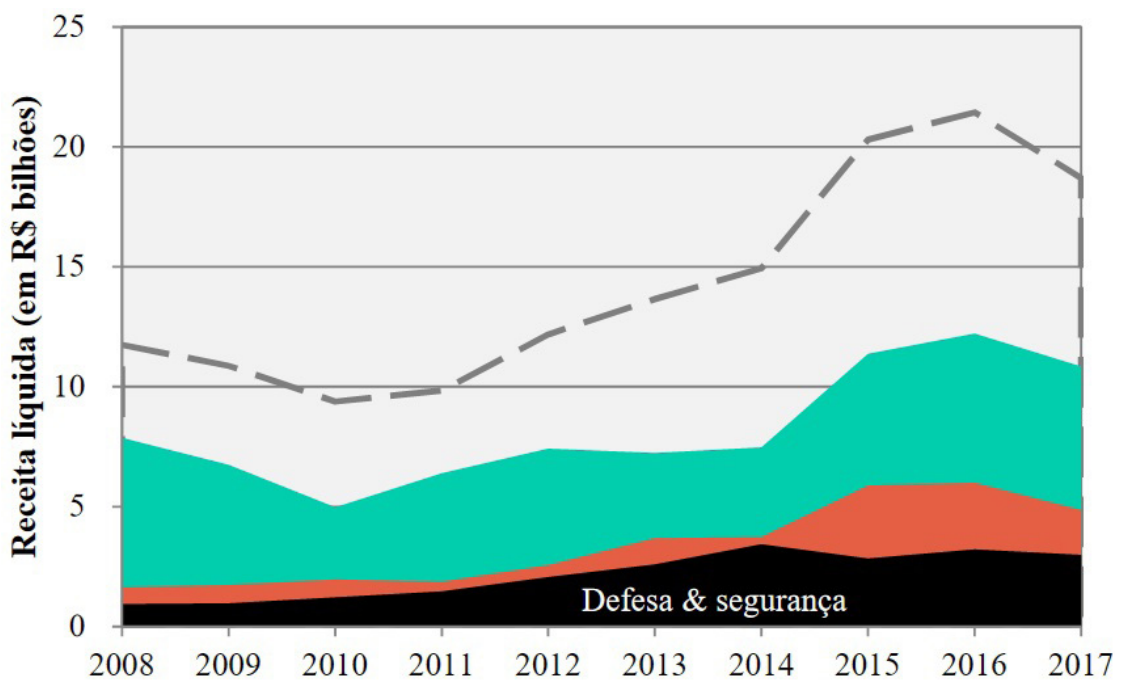

Figura 4. Faturamento da Embraer: receita líquida gerada ao longo da última década. Adaptado de: Relatórios Anuais de 2008-2017 [8]. 
A robustez desses valores é um indicativo da necessidade de pesquisas no país em novas tecnologias para aplicação no setor aeronáutico, como as de união de materiais no estado sólido. No entanto, existem poucos estudos no Brasil demonstrando a viabilidade do FSpW. Campanelli et al. [9] reportaram em seu estudo a otimização dos parâmetros de processo considerando a resistência ao cisalhamento de chapas da liga de magnésio AZ31 com $2 \mathrm{~mm}$ de espessura. No campo de união de materiais poliméricos, Gonçalves et al. [10] estudaram a otimização dos parâmetros de processo envolvidos na soldagem a ponto por fricção de chapa de poliamida 6 e laminado de poliamida 66 com fibra de carbono. No entanto, não é do nosso conhecimento a publicação de nenhum trabalho anterior relacionado com a otimização de parâmetros de processo para a soldagem a ponto por fricção de chapas finas de ligas de alumínio para aplicação aeronáutica até o presente momento.

Este estudo tem como objetivo a otimização da condição de soldagem a ponto por fricção de chapas de 1,6 mm da liga AA2198-T8 sobrepostas com base no método Taguchi para Planejamento de Experimentos, através da investigação de parâmetros importantes para o processo - velocidade de rotação, tempo de soldagem e profundidade de penetração - e sua relação com a resistência mecânica das juntas quando solicitadas em cisalhamento.

\section{Materiais e Métodos}

Neste estudo, foram avaliadas juntas sobrepostas de chapas com $1.6 \mathrm{~mm}$ de espessura da liga do sistema Al-Cu-Li AA2198 tratada termicamente sob a condição T8 (solubilização, trabalho a frio e envelhecimento artificial). A soldagem a ponto por fricção foi realizada no equipamento RPS 100, e a variante do processo utilizada foi a penetração da camisa com uma força axial constante de $14,6 \mathrm{kN}$. Os corpos de prova para os testes de resistência ao cisalhamento das juntas soldadas pela sobreposição das chapas foram produzidos com base nas dimensões previstas pela norma ISO 14273 [11], os quais foram posteriormente testados em uma máquina universal de ensaios Zwick-Roell 1478 com velocidade de deslocamento constante de $1 \mathrm{~mm} / \mathrm{s}$.

Para a determinação da condição de soldagem otimizada e avaliação dos efeitos da variação dos parâmetros de processos - velocidade de rotação (VR), tempo de soldagem (TS) e profundidade de penetração (PP) - foi empregada a metodologia de Planejamento de Experimentos (DOE) Taguchi, considerando-se como variável de resposta a carga de cisalhamento (CC). Esta metodologia permite, com alta eficiência, a identificação dos efeitos principais associados a cada um dos fatores através de uma matriz de experimentos reduzida, ainda que interação entre os fatores não seja considerada [12]. Com o objetivo de analisar os três fatores apresentados, uma matriz L9 foi empregada, resultando em nove experimentos - três fatores com três níveis associados a cada um. Os experimentos foram realizados em triplicata.

A janela de parâmetros (Tabela 1) para o desenvolvimento da análise foi determinada com base em características como ausência de defeitos volumétricos nas juntas e resistência mecânica, além a revisão de trabalhos anteriores disponíveis na literatura $[13,14]$.

Tabela 1. Parâmetros de processo da soldagem a ponto por fricção: fatores e seus níveis.

\begin{tabular}{lccc}
\multicolumn{1}{c}{ Fator } & Nível 1 & Nível 2 & Nível 3 \\
Velocidade de rotação $[\mathrm{rpm}]$ & 1100 & 1300 & 1500 \\
Tempo de soldagem [s] & 3 & 4 & 5 \\
Profundidade de penetração $[\mathrm{mm}]$ & 2,1 & 2,6 & 2,8 \\
\hline
\end{tabular}

\section{Resultados e Discussão}

\subsection{Método Taguchi - otimização dos parâmetros}

A matriz de experimentos L9 obtida através da metodologia Taguchi com as condições de soldagem considerando os três fatores propostos - velocidade de rotação (VR), tempo de soldagem (TS) e profundidade de penetração (PP) - pode ser observada na Tabela 2, juntamente com as médias das cargas de cisalhamento obtidas através do ensaio mecânico dos corpos de prova. Observa-se que a combinação de parâmetros associada à maior resistência ao cisalhamento é a C7 (1500 rpm, $3 \mathrm{~s}, 2,8 \mathrm{~mm})$, com $7947 \mathrm{~N}$, que corresponde à condição com os maiores níveis de VR e PP. No outro extremo, a menor carga de cisalhamento média foi encontrada na condição 
Tabela 2. Condições experimentais (velocidade de rotação, tempo de soldagem e profundidade de penetração) e as cargas de cisalhamento média [N] correspondentes.

\begin{tabular}{ccccc}
\hline Condição de soldagem & VR [rpm] & TS $[\mathbf{s}]$ & PP [mm] & Carga de cisalhamento média [N] \\
C1 & 1100 & 3 & 2,1 & $4877 \pm 78$ \\
C2 & 1100 & 4 & 2,6 & $5733 \pm 270$ \\
C3 & 1100 & 5 & 2,8 & $7685 \pm 420$ \\
C4 & 1300 & 3 & 2,6 & $5799 \pm 110$ \\
C5 & 1300 & 4 & 2,8 & $6996 \pm 747$ \\
C6 & 1300 & 5 & 2,1 & $5650 \pm 505$ \\
C7 & 1500 & 3 & 2,8 & $7947 \pm 349$ \\
C8 & 1500 & 4 & 2,1 & $7426 \pm 724$ \\
C9 & 1500 & 5 & 2,6 & $7498 \pm 237$ \\
\hline
\end{tabular}

C1 (1100 rpm, 3 s, 2,1 mm - 4877 N), cujos fatores apresentam os menores níveis de VR e PP, em oposição àqueles encontradas na C7. Todas as condições de soldagem testadas neste experimento, no entanto, excedem o valor mínimo requerido pela norma AWS D17.2 [15] para aplicações de soldas a ponto por resistência em estruturas aeronáuticas, correspondente a $3180 \mathrm{~N}$ para o material estudado.

Os gráficos dos efeitos principais da análise Taguchi (apresentados na Figura 5) permitem prever o comportamento da variação da carga de cisalhamento em função da variação de parâmetros do processo, bem como a previsão da combinação de parâmetros que proporcionaria a melhor resposta buscada. A condição otimizada indicada pela ferramenta estatística corresponde à combinação de VR = $1500 \mathrm{rpm}, \mathrm{TS}=5 \mathrm{~s}$ e PP = 2,8 mm, que foi identificada como C10 uma vez que os parâmetros não compõem a matriz L9 com os experimentos iniciais. A Tabela 3 mostra a média dos resultados do ensaio das soldas da nova condição, onde se observa que o valor da carga de cisalhamento média (7759 N) não excede o valor máximo da CC encontrado previamente no experimento - atribuído à condição C7 (7949 N). Além disso, a condição C7, que possui os níveis de VR e PP idênticos à condição otimizada e difere apenas no TS, apresenta-se mais vantajosa também pelo menor tempo de soldagem ( 3 s) em relação a C10 (5 s),

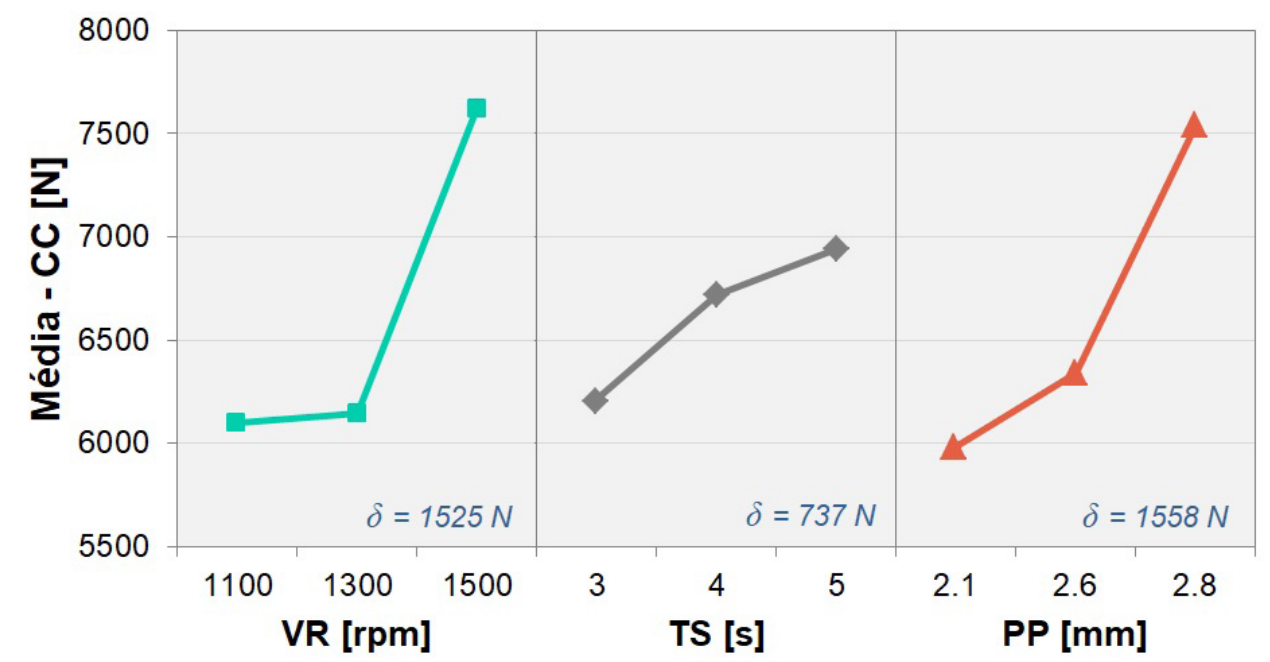

Figura 5. Efeitos principais da análise Taguchi da matriz L9 para a média da carga de cisalhamento.

Tabela 3. Combinação de parâmetros otimizada (velocidade de rotação (VR), tempo de soldagem (TS) e profundidade de penetração (PP)) indicada pelo Taguchi para a maximização da carga de cisalhamento média.

\begin{tabular}{ccccc}
\hline Condição de soldagem & VR $[\mathrm{rpm}]$ & TS $[\mathbf{s}]$ & PP $[\mathrm{mm}]$ & Carga de cisalhamento média [N] \\
C10 & 1500 & 5 & 2,8 & $7759 \pm 428$ \\
\hline
\end{tabular}


uma vez que menores ciclos de soldagem possibilizam uma maior taxa de produtividade dessas juntas - o que é bastante desejável tendo em vista aplicações industriais, em especial considerando-se o volume de produção associado ao setor automobilístico.

Além da indicação da condição ótima de soldagem prevista pelo método, a Figura 5 mostra o efeito da variação de cada um dos parâmetros na variável resposta (CC). É possível observar que diferença entre a máxima e mínima carga de cisalhamento (delta - $\delta$ ) relativa à variação dos níveis de $3 \mathrm{~s}$ a $5 \mathrm{~s}$ do fator TS é de apenas $737 \mathrm{~N}$, o que indica uma influência menor deste fator na resistência mecânica das juntas. Nota-se ainda, que a partir de $4 \mathrm{~s}$ o aumento do tempo de soldagem produz uma resposta cada vez menos significante na CC, evidenciada pela menor inclinação da curva comparada ao intervalo de 3-4 s. Os fatores VR e PP, por outro lado, são notadamente mais relevantes na variação da carga de cisalhamento: a variação dos níveis de 1100-1500 rpm é associada a um delta de $1525 \mathrm{~N}$ na CC, enquanto o aumento da profundidade de penetração da camisa de 2,1 a 2,8 mm produz uma variação de $1558 \mathrm{~N}$ na variável de resposta. Além disso, o comportamento de ambos os fatores na variação da média da carga de cisalhamento é bastante similar: ao contrário do observado no comportamento da variação dos níveis do TS, a variação progressiva da VR e do PP produz uma resposta na variação da CC cada vez maior, especialmente na segunda fração das curvas. $O$ aumento considerável na inclinação das curvas entre os níveis 2 e 3 indica que os efeitos da variação dos parâmetros é mais acentuado quanto maior for a VR e PP associados ao processo. Ou seja, quanto maiores VR e PP, maior a média da carga de cisalhamento das juntas.

\subsection{ANOVA - Análise da variância}

A análise da variância (ANOVA) dos efeitos individuais dos parâmetros na carga de cisalhamento (apresentada na Tabela 4 e Figura 6) confirma que a VR e PP são os fatores principais de influência na CC, correspondendo juntos a $86 \%$ de contribuição na variável de resposta. Embora os resultados indiquem que a VR apresenta $46 \%$ da influência

Tabela 4. Análise da variância e influência individual dos parâmetros (velocidade de rotação (VR), tempo de soldagem (TS) e profundidade de penetração (PP)) na carga de cisalhamento média.

\begin{tabular}{cccccc}
\hline Fator & GL & SQ & MQ & F & \% I \\
VR & 2 & 4505750 & 2252875 & 8,33 & 46 \\
TS & 2 & 854534 & 427267 & 1,58 & 9 \\
PP & 2 & 540734 & 1997842 & 7,39 & 40 \\
Erro & 2 & 9896702 & 270367 & - & 5 \\
\hline
\end{tabular}

$\mathrm{GL}=$ Graus de liberdade, $\mathrm{SQ}$ = soma dos quadrados, $\mathrm{MQ}=$ média dos quadrados, \% I = influência (porcentagem).

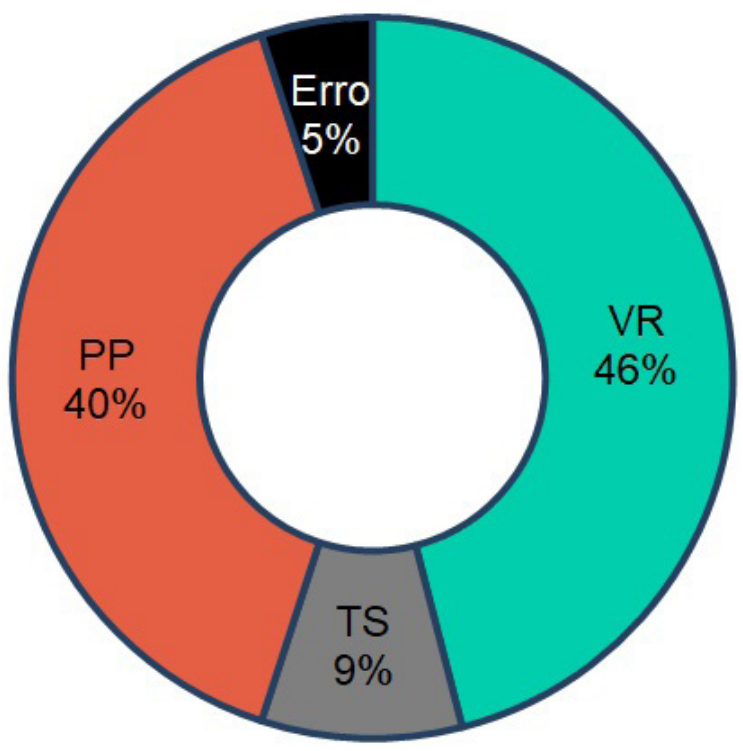

Figura 6. ANOVA: influência dos parâmetros individuais na variância da média da carga de cisalhamento. 
na variação da CC, não é possível afirmar que ela é isoladamente a maior contribuinte para a resistência das juntas uma vez que o erro da análise deve ser considerado (5\%), o que coloca a PP estatisticamente tão significante quanto à rotação da ferramenta. Desta forma, a VR e PP são os fatores mais importantes que controlam a carga de cisalhamento das soldas. Um estudo de soldas a ponto por fricção dissimilares de chapas de AA6168-T8 e Ti-6Al-4V realizado por Plaine et al. [6] e um trabalho publicado por Kubit et al. [16] sobre RFSSW de chapas de AA7975 com $0,8 \mathrm{~mm}$ de espessura concluíram que a VR é responsável pela maior porcentagem de contribuição na variação da CC. Outros autores, no entanto, reportam que o fator mais importante para a propriedade mecânica das juntas é a PP, como Campanelli et al. [9] em um estudo sobre a RFSSW de chapas da liga de magnésio AZ31.

Ademais, a análise da variância atribui ao tempo de soldagem uma porcentagem de influência consideravelmente menos expressiva para a carga de cisalhamento das juntas, especialmente quando comparada com o erro - $9 \%$ para o TS contra $5 \%$ de \% do erro. A contribuição minoritária do TS foi encontrada em outros estudos similares envolvendo RFSSW de ligas leves: Campanelli et al. [9] também reporta em seu trabalho sobre a soldagem de ligas de magnésio com $2 \mathrm{~mm}$ de espessura que a porcentagem de influência do tempo de soldagem é significativamente menor que o erro (4,38\% para o TS contra $16,58 \%$ para o erro), o que permite concluir que a variável de processo é estatisticamente irrelevante para a carga de cisalhamento das juntas em pequenas faixas de variações.

\subsection{Efeitos metalúrgicos da variação dos parâmetros}

A análise da variância dos parâmetros de processo em relação à resistência mecânica das juntas indicou que a velocidade de rotação e profundidade de penetração são majoritariamente responsáveis pela variação dos efeitos metalúrgicos que determinam as propriedades mecânicas das soldas.

A Figura 7 mostra uma macrografia representativa da seção transversal de uma solda do experimento. É possível observar a microestrutura com grãos pequenos e equiaxiais da zona de mistura (região do botão de solda), característica do processo de recristalização dinâmica. A velocidade de rotação da ferramenta durante a soldagem está diretamente relacionada à taxa de deformação do material: quando maior a VR, maior a taxa. Além disso, a VR também interfere na geração de calor através da fricção da ferramenta com o material durante o processo. Tomando-se a geração da energia térmica como consequência do torque, o aporte de calor pode ser definido como apresentado na Equação 1

$$
Q_{a p p}=\sum_{n=1}^{n} T \omega \Delta t
$$

em que $Q_{\text {app }}$ é a energia térmica resultante do processo, T é o torque associado à ferramenta, $\omega$ é velocidade angular da ferramenta (ou VR) e $\Delta$ t é o tempo associado à duração do processo, conforme apresentado por Tier et al. [17]. Os efeitos da variação da VR na qualidade das juntas estão diretamente relacionados ao fenômeno de recristalização dinâmica determinante na microestrutura do botão de solda, uma vez que maiores valores de VR causam maior deformação e geração de calor. A VR também é determinante para a plasticização e mistura do material, já que o maior aporte de calor e taxa de deformação gerados pela elevação da velocidade da ferramenta são responsáveis pela diminuição da viscosidade do material, como demonstraram Franke et al. [18].
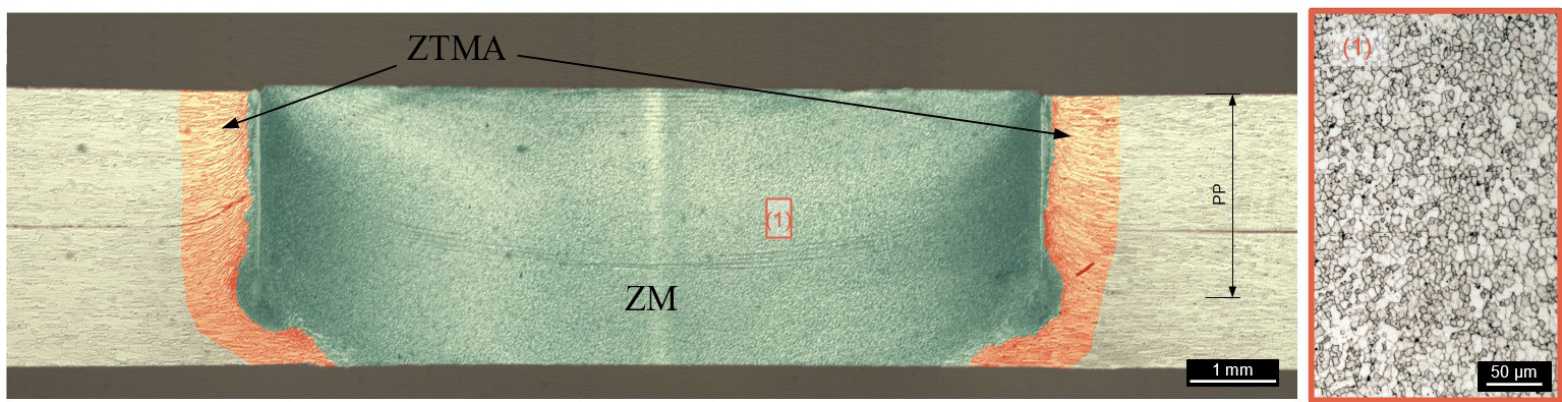

Figura 7. Seção transversal de uma solda a ponto por fricção com a zona de mistura (ZM) e zona termo-mecanicamente afetada (ZTMA) identificadas. Destaque para a microestrutura da ZM à direita. 
O outro parâmetro responsável pela variação da carga de cisalhamento juntamente com a VR, a profundidade de penetração da camisa é a variável determinante na fração do material da chapa que participa da soldagem, como pode ser observado na Figura 7. De Castro et al. [19] mostrou a relação entre a área da zona de mistura e a resistência ao cisalhamento da soldagem de chapas de AA2198 com 3,2 mm de espessura: quanto maior a área da ZM da solda, maior a carga em cisalhamento suportada. Além disso, o estudo mostra que maiores valores de PP facilitam a propagação de trincas circunferenciais e dificulta a propagação da componente anular da trinca através da zona de mistura, frequentemente responsável pela fratura das peças soldadas, já que maiores penetrações geram uma maior interface entre o botão de solda e o material base.

\section{Conclusão}

A otimização do processo de soldagem a ponto por friç̧ão de chapas de alumínio AA2198-T8 com 1,6 mm de espessura foi estudada com base em ferramentas estatísticas. Através dos resultados e análises, foi possível concluir que todas as soldas produzidas com base na combinação de parâmetros da matriz L9 do método Taguchi apresentaram resistência ao cisalhamento superior ao requerido pela norma AWS D17.2 (3180 N) para aplicação aeronáutica, com cargas em cisalhamento variando de $4877 \mathrm{~N}$ a $7947 \mathrm{~N}$. Além disso, para a janela de parâmetros selecionada, as duas ferramentas estatísticas aplicadas no estudo indicaram que a VR e PP são os parâmetros que mais influenciam na carga de cisalhamento das soldas. Os consideravelmente maiores valores de delta obtidos através dos gráficos dos efeitos principais da análise Taguchi para a VR e PP, comparados ao TS, são indicativos da maior relevância desses dois parâmetros, o que é confirmado através da análise da variância (ANOVA) que aponta que VR e PP influenciam $46 \%$ e $40 \%$, respectivamente, da variação nos valores de carga de cisalhamento das juntas. Em contraste, a ANOVA mostrou que a influência do TS é expressivamente menos significativa para a carga de cisalhamento das juntas.

Por fim, com base em critérios econômicos e de eficiência necessários para aplicações industriais, encontrou-se que a condição de parâmetros otimizada é a condição C7 (VR $=1500 \mathrm{rpm}, \mathrm{TS}=3 \mathrm{~s}, \mathrm{PP}=2,8 \mathrm{~mm}$ ), mesmo que a análise do método Taguchi tenha previsto a condição $\mathrm{C} 10$ (VR $=1500 \mathrm{rpm}, \mathrm{TS}=5 \mathrm{~s}, \mathrm{PP}=2,8 \mathrm{~mm}$ ) como ótima. Além da condição C7 apresentar valor da média da carga de cisalhamento superior ao da C10 (7949 N contra 7759 N), o menor tempo de soldagem associado a essa combinação de parâmetros é mais atrativo industrialmente visando menores ciclos de soldagem e maior produtividade, em especial para o setor automobilístico.

\section{Agradecimentos}

Os autores agradecem à CAPES (código de financiamento 001) e ao CNPq pelo apoio financeiro (processo no. 134520/2017-3), e também ao Helmoltz Zentrum Geesthacht (HZG) por viabilizar este estudo.

\section{Referências}

[1] Amancio-Filho ST, Camillo AP, Bergmann L, Dos Santos JF, Kury SE, Machado NG. Preliminary investigation of the microstructure and mechanical behaviour of 2024 aluminium alloy friction spot welds. Materials Transactions. 2011;52(5):985-991. http:// dx.doi.org/10.2320/matertrans.L-MZ201126.

[2] Lage SBM. Otimização dos parâmetros de soldagem a ponto por fricção (FSpW) da liga AIMgSc e avaliação das propriedades mecânicas estáticas e dinâmicas [dissertação de mestrado]. São Carlos: Universidade Federal de São Carlos; 2018.

[3] Rioja RJ, Liu J. The evolution of Al-Li base products for aerospace and space applications. Metallurgical and Materials Transactions. A, Physical Metallurgy and Materials Science. 2012;43(9):33253337. http://dx.doi.org/10.1007/s11661-012-1155-z.

[4] Yang C-W, Hung F-Y, Lui T-S, Chen L-H, Juo J-Y. Weibull Statistics for evaluating failure behaviors and joining reliability of friction stir spot welded 5052 aluminum alloy. Materials Transactions. 2009;50(1):145-151. http://dx.doi.org/10.2320/matertrans. MRA2008341.
[5] Silva A, Tier M, Bergman L, Rosendo T, Ramos F, Mazzaferro J, Mazzaferro C, Strohaecker T, Santos J. Friction spot and friction stir spot welding processes - a literature review. Bulletin of National R\&D. 2007;3:36-44.

[6] Plaine AH, Gonzalez AR, Suhuddin UFH, Santos JF, Alcântara NG. The optimization of friction spot welding process parameters in AA6181-T4 and Ti6Al4V dissimilar joints. Materials \& Design. 2015;83:36-41. http://dx.doi.org/10.1016/j.matdes.2015.05.082.

[7] Mishra RS, MaZY. Friction stir welding and processing. Materials Science and Engineering R Reports. 2005;50(1-2):1-78. http:// dx.doi.org/10.1016/j.mser.2005.07.001.

[8] EMBRAER. Relatórios anuais de 2008 a 2017 [página de internet]. [acesso em 28 ago. 2018]. Disponível em: https://ri.embraer. com.br/show.aspx?idCanal=FQxR65+10mnFsUzhlziJFQ==

[9] Campanelli LC, Suhuddin UFH, Santos JF, Alcântara NG. Parameters optimization for friction spot welding of AZ31 magnesium alloy by Taguchi method. Soldagem e Inspeção. 2012;17(1):26-31. http://dx.doi.org/10.1590/S0104-92242012000100005. 
[10] Gonçalves J, Santos JF, Canto LB, Amancio ST Fo. Aperfeiçoamento da técnica de Soldagem Pontual por Friç̧ão ( $F S p W)$ para união de poliamida 6 e laminado de poliamida 66 com fibra de carbono. Soldagem e Inspeção. 2014;19(1):19-27. http:// dx.doi.org/10.1590/S0104-92242014000100004.

[11] International Organization for Standardization. ISO 14273:2016 - Resistance welding - Destructive testing of welds - Specimen dimensions and procedure for tensile shear testing resistance spot and embossed projection welds. Geneva: ISO; 2016.

[12] Ulrich KT, Eppinger SD. Product design and development. New York: McGraw-Hill Education; 2015.

[13] Pieta G, Santos J, Strohaecker TR, Clarke T. Optimization of friction spot welding process parameters for AA2198-T8 sheets. Materials and Manufacturing Processes. 2014;29(8):934-940. http://dx.doi.org/10.1080/10426914.2013.811727.

[14] Sree Sabari S, Malarvizhi S, Balasubramanian V. Characteristics of FSW and UWFSW joints of AA2519-T87 aluminium alloy: effect of tool rotation speed. Journal of Manufacturing Processes. 2016;22:278-289. http://dx.doi.org/10.1016/j.jmapro.2016.03.014.

[15] American Welding Society. AWS D17.2/D17.2M:2013-Specification for Resistance Welding for Aerospace Applications. Miami: AWS; 2013.
[16] Kubit A, Kluz R, Trzepieciński T, Wydrzyński D, Bochnowski W. Analysis of the mechanical properties and of micrographs of refill friction stir spot welded 7075-T6 aluminium sheets. Archives of Civil and Mechanical Engineering. 2018;18(1):235-244. http:// dx.doi.org/10.1016/j.acme.2017.07.005.

[17] Tier MD, Rosendo TS, Santos JF, Huber N, Mazzaferro JA, Mazzaferro $\mathrm{CP}$, et al. The influence of refill FSSW parameters on the microstructure and shear strength of 5042 aluminium welds. Journal of Materials Processing Technology. 2013;213(6):9971005. http://dx.doi.org/10.1016/j.jmatprotec.2012.12.009.

[18] Franke DJ, Morrow JD, Zinn MR, Duffie NA, Pfefferkorn FE. Experimental determination of the effective viscosity of plasticized aluminum alloy 6061-T6 during friction stir welding. Procedia Manufacturing. 2017;10:218-231. http://dx.doi.org/10.1016/j. promfg.2017.07.050.

[19] Castro CC, Plaine AH, Dias GP, Alcântara NG, Santos JF. Investigation of geometrical features on mechanical properties of AA2198 refill friction stir spot welds. Journal of Manufacturing Processes. 2018;36(2018):330-339. http://dx.doi.org/10.1016/j. jmapro.2018.10.027. 